\title{
PERCEPCIÓN DE TURISTAS NACIONALES Y FORÁNEOS DEL SERVICIO EN RESTAURANTES DE TODOS SANTOS PUEBLO MÁGICO, MÉXICO
}

\section{PERCEPTION OF NATIONAL AND FOREIGN TOURISTS OF THE SERVICE IN RESTAURANTS FROM TODOS SANTOS MAGICAL TOWN, MEXICO}

\author{
Mauro Alejandro Monroy Ceseña \\ Universidad Autónoma de Baja California Sur \\ México \\ monroym@uabcs.mx
}

\author{
Francisco Javier Urcádiz Cázares \\ Instituto Tecnológico Nacional de México \\ Campus La Paz, México \\ urcadiz@me.com
}

Fecha de recepción: 24/12/2019 - Fecha de aprobación: 12/04/2020

DOI: https://doi.org/10.36995/j.visiondefuturo.2020.24.02.006.es

\section{RESUMEN}

El pueblo mágico de Todos Santos es un sitio estratégico para el desarrollo local y dependiente de la economía turística incentivada por visitantes nacionales y foráneos. Los restaurantes del pueblo obtienen la materia prima de la región para prestar servicios en diferentes especialidades de comida. La calidad del servicio (SQ) y la satisfacción del cliente (CS) son dos constructos que al evaluarse permiten potenciar las estrategias de cualquier mercado. En este estudio, se evalúa la percepción y la relación de la SQ y la CS en las diferentes especialidades de restaurantes contrastando la percepción entre los turistas nacionales y foráneos. Mediante 162 encuestas se valoraron los constructos utilizando cinco dimensiones: instalaciones, accesibilidad, capital humano, atmósfera y alimentos, donde hipotéticamente la de alimentos y capital humano deberían ser los principales determinantes. Los resultados sugieren que la SQ y la CS se perciben de manera similar entre ambos segmentos, excepto en la especialidad Italiana en todas sus dimensiones y parcialmente la Japonesa. La dimensión de comida fue la mayor valorada, sin embargo, no fue significativamente diferente del resto de las dimensiones. La SQ tuvo un impacto en la CS dada la percepción de los comensales por segmento de acuerdo a un análisis de correlación, cuyo efecto causal deber ser profundizado.

PALABRAS CLAVE: Calidad, Percepción, Restaurantes, Servicio, Satisfacción.

"Visión de Futuro" Año 17, Volumen No 24 N², Julio - Diciembre 2020 - Pág 194 - 210 URL de la Revista: http://visiondefuturo.fce.unam.edu.ar/index.php/visiondefuturo/index

URL del Documento: https://visiondefuturo.fce.unam.edu.ar/index.php/visiondefuturo/issue/view/18 ISSN 1668 - 8708 - Versión en Línea 


\section{ABSTRACT}

The magic town of Todos Santos is a strategic site for local development and is dependent on the tourist economy driven by national and foreign visitors. Restaurants in Todos Santos obtain raw materials locally to provide quality products for different food specialties. The quality of service (SQ) and customer satisfaction (CS) are two constructs that when evaluated are critical for any market. In this study, the perception and relationship between SQ and SC in the different restaurant specialties is evaluated, contrasting the perception between national and foreign tourists segments. Using 162 surveys, the constructs were evaluated using five dimensions: installations, accessibility, human capital, atmosphere, and food, where hypothetically food and human capital should be the main determinant. The results suggest that SQ and CS are perceived similarly between both segments, except in the Italian specialty in all its dimensions, and partially in Japanese. The food dimension was the highest value on average however, it was not significantly different from the rest of the dimensions. The SQ had a moderate positive impact on CS given the perception of diners by segment based on correlation analysis, the causal effect of which must be determined with detail.

KEYWORDS: Quality, Perception, Restaurants, Service, Satisfaction.

\section{INTRODUCCIÓN}

Los pueblos mágicos son sitios socio-estratégicos promovidos por el gobierno federal mexicano para desarrollar la economía a través del turismo sostenible y cultural, con énfasis en el valor del patrimonio local (Rodríguez-Herrera et al., 2018). Esta política implica el desarrollo de pequeños establecimientos de restaurantes y de servicios hoteleros. Todos Santos es un pueblo mágico ubicado al sur de la península de Baja California Sur (México) y es reconocido por un microclima que contrasta con las condiciones áridas prevalentes en la región. Esto permite, por un lado, el cultivo especial de una variedad de vegetales para un clima templado y tropical, y debido a su condición costera, los residentes tienen acceso a los recursos marinos como pecados y mariscos. Estos recursos son fuentes primarias para varios establecimientos que ofrecen un servicio de restaurante. Durante el verano, las visitas de turistas nacionales y

\footnotetext{
"Visión de Futuro" Año 17, Volumen No 24 N² 2, Julio - Diciembre 2020 - Pág 194 - 210

URL de la Revista: http://visiondefuturo.fce.unam.edu.ar/index.php/visiondefuturo/index

URL del Documento: https://visiondefuturo.fce.unam.edu.ar/index.php/visiondefuturo/issue/view/18

ISSN 1668 - 8708 - Versión en Línea

E-mail: revistacientifica@fce.unam.edu.ar
} 
foráneos aumentan en el pueblo, incrementado el consumo en los restaurantes locales. El consumo de turistas en los restaurantes es esencial para sostener la economía local, por lo tanto, la evaluación del servicio debe ser parte de las estrategias comerciales. Sin embargo, la calidad de servicio percibida (SQ) y la satisfacción del cliente (CS) son dos constructos fundamentales que permanecen sin explorar en la localidad.

En el sector económico de los restaurantes, la percepción de la SQ y los juicios sobre la CS son relevantes para los tomadores de decisiones (Ryu, Lee y Gon-Kim, 2012; GuzmánLópez y Cárcamo-Solís, 2014; Vera y Trujillo, 2017; Kim, Seo y Nurhidayati, 2019), debido a que conducen a un perfil deseable empresarial a partir de las opiniones de los comensales (Kotler y Armstrong, 2012). Los clientes al experimentar un servicio, mantienen un juicio de valor en función de las diferentes formas de percibir y sentir durante un proceso (MolinerCantos, 2001), de esta manera se forman una opinión sobre las empresas, sus productos y sus servicios. Finalmente, los clientes responden hacia la lealtad de un restaurante y la recomendación del mismo, generando un impacto positivo en la empresa. El análisis sistemático de la SQ y de la CS permite evaluar desde una perspectiva estratégica a los factores que impactan benéficamente a una organización (Armstrong, 2012).

La relación entre la $S Q$ y la $C S$ ha desarrollado una enorme atención dentro de la literatura de marketing (Gacic et al., 2013; Muhamad, Hashim y Rozila, 2016; Monroy-Ceseña \& Urcádiz-Cázares, 2019; Nguyen et al., 2019; Sartor, 2019) por su relación cercana y posiblemente directa. Algunos autores consideran que la SQ tiene un impacto en la CS (Aeker \& Jacobson, 1994; Gilbert et al., 2004; Gilbert \& Veloutsou, 2006; Zárraga et al., 2018) y ambas construcciones deben evaluarse para que las empresas reconozcan el servicio desde la perspectiva del cliente.

La SQ y la CS son dos índices de alta relevancia que contribuyen al posicionamiento en el mercado y ayudan a generar mayores ganancias en cualquier mercado. En este sentido, el mercado de restaurantes turísticos del pueblo mágico de Todos Santos establece un escenario sin precedentes para la evaluación y la comparación tanto de la SQ como de la CS. El propósito de este trabajo es evaluar las percepciones de los comensales sobre la SQ y la CS en los restaurantes durante el verano de 2018, comparando al segmento de turistas nacionales y foráneos de acuerdo a la especialidad de los restaurantes.

\footnotetext{
"Visión de Futuro" Año 17, Volumen No 24 N 2, Julio - Diciembre 2020 - Pág 194 - 210

URL de la Revista: http://visiondefuturo.fce.unam.edu.ar/index.php/visiondefuturo/index

URL del Documento: https://visiondefuturo.fce.unam.edu.ar/index.php/visiondefuturo/issue/view/18

ISSN 1668 - 8708 - Versión en Línea

E-mail: revistacientifica@fce.unam.edu.ar
} 


\section{DESARROLLO}

\section{Antecedentes y marco teórico}

\section{Calidad del servicio}

La calidad del servicio (SQ) es una construcción que ha evolucionado en su definición y ha sido estudiada de varias maneras, sin embargo, es un punto de referencia a nivel empresarial (Armstrong, 2012). El uso diverso de múltiples dimensiones incluidas en los constructos impide una definición concisa. La definición multidimensional de la SQ (Buttle, 1996; Ladhari, 2009) ha sido ampliamente utilizada y adaptada a diferentes industrias (Fujun et al., 2007; Ladhari, 2008, 2009) dadas las características particulares de cada negocio y cada región. El trabajo de Cronin \& Taylor, (1994) ha sido un referente a nivel mundial, donde se define a la calidad como una forma de actitud que está relacionada a la satisfacción, y se origina al hacer comparaciones de las expectativas con las percepciones de calidad cuando un cliente tiene una experiencia de servicio (Akbaba, 2006; Wilkins et al., 2007). La calidad percibida del servicio puede ser utilizada a nivel estratégico por las empresas, de tal manera que contribuya a la estructura y funcionalidad a nivel organizacional de acuerdo a los factores que los clientes consideran entre sus expectativas, percepciones y necesidades.

Sobre los estudios observacionales en el sector de restaurantes, algunas dimensiones como los alimentos y el capital humano han sido percibidas como el factor principal. Namin (2017), cita en su investigación que el factor determinante en la calidad del servicio percibido por los comensales es la comida. Según sus principales hallazgos, la oferta de un menú saludable y acompañado de alimentos sabrosos se convierten en criterios impactantes en la búsqueda de la satisfacción de los comensales en esa industria. Por otro lado, la dimensión del capital humano sirve como un factor preponderante para garantizar la calidad del servicio ofrecido según Hernándes-de-Velazco, Chumaceiro, \& Atencio_(2009) y Ryu \& Han (2011). En contraste, Voon (2012) argumenta de acuerdo a su estudio sobre la calidad del servicio en restaurantes de comida rápida, que uno de los principales factores que influyen en la percepción del cliente es la influencia del capital humano en su interacción continua con los clientes, así como el precio de venta asignado al suministro de alimentos.

\footnotetext{
“Visión de Futuro" Año 17, Volumen No 24 No 2, Julio - Diciembre 2020 - Pág 194 - 210

URL de la Revista: http://visiondefuturo.fce.unam.edu.ar/index.php/visiondefuturo/index

URL del Documento: https://visiondefuturo.fce.unam.edu.ar/index.php/visiondefuturo/issue/view/18

ISSN 1668 - 8708 - Versión en Línea

E-mail: revistacientifica@fce.unam.edu.ar
} 


\section{La satisfacción del cliente}

Al revisar la literatura sobre la satisfacción del cliente (CS), es posible encontrar varios enfoques, uno de ellos considera la satisfacción como el resultado o la respuesta de la experiencia de consumo. Autores como Westbrook y Reilly (1987) definen la CS como una respuesta emocional a las experiencias relacionadas con la compra de ciertos productos y servicios. Los sentimientos de placer y la satisfacción de las expectativas provocan un estado de satisfacción en los comensales, esto conduce a la lealtad del consumo (Zairi, 2000; Namkung, Cheong y Choi, (2011) y, como proceso, trae consigo un juicio de valor que subyace a la experiencia de consumir (Yi, 1991).

Autores como Spreng y Olshavsky (1993); Spreng, MacKenzie y Olshavsky (1996) consideran a la satisfacción como una respuesta posterior a la compra (que iguala o excede los deseos del consumidor) ya que es una sensación que se deriva una vez que el consumo se hace frente a las recompensas, los costos y su consecuencia esperada. Del mismo modo, Mano y Oliver (1993) definen SC como una actitud y juicio evaluativo post-consumo que varía a lo largo de un continuo hedónico.

Otra perspectiva considera a la CS como una transacción específica o acumulativa. Según el primer enfoque, autores como Halstead, Hartman y Schmidt (1994), Evrard y Aurier (1996) afirman que la CS se centra en las respuestas de los clientes ante una transacción particular de un producto o de un servicio, estableciendo un estado psicológico resultante de la experiencia de compra y consumo (post-elección). En el segundo enfoque, la satisfacción se asocia con una transacción acumulativa donde el juicio del consumidor implica una evaluación global de las experiencias a lo largo del tiempo, es decir, una historia de las experiencias individuales ya sean satisfactorias o insatisfactorias (Vanhamme; 2002; Aurier, Evrard \& N'Goala 2004).

Aunque el constructo de la CS se utiliza ampliamente, su definición no es concisa (Oliver, Rust y Varki, 1997) e implica una variación dentro de los instrumentos de medición (Gardial et al., 1994; Yi, 1991). Sin embargo, la CS es una parte esencial del marketing y juega un papel fundamental en la toma de decisiones en el mercado (Oliver, Rust \& Varki, 1997). En este sentido, si las empresas tienen clientes satisfechos con su oferta de servicios, podrían mantener un nivel de competitividad y persistir o mejorar en el mercado.

\footnotetext{
"Visión de Futuro" Año 17, Volumen No 24 N 2, Julio - Diciembre 2020 - Pág 194 - 210

URL de la Revista: http://visiondefuturo.fce.unam.edu.ar/index.php/visiondefuturo/index

URL del Documento: https://visiondefuturo.fce.unam.edu.ar/index.php/visiondefuturo/issue/view/18

ISSN 1668 - 8708 - Versión en Línea

E-mail: revistacientifica@fce.unam.edu.ar
} 


\section{Objetivo e hipótesis}

El objetivo de este trabajo es evaluar las percepciones de los comensales sobre la SQ y la CS en los restaurantes, comparando turistas (en adelante comensales) nacionales y foráneos, de acuerdo con la especialidad alimentaria de los restaurantes.

En particular, esta investigación propone las siguientes hipótesis:

H1: Los promedios de la SQ y la CS percibidos por los comensales nacionales difieren de los foráneos, según las especialidades de los restaurantes.

H2: Las dimensiones de los alimentos y el capital humano son los principales determinantes en la SQ y/o la CS en la percepción del restaurante para cada segmento (comensales nacionales y foráneos).

H3: Los promedios de la SQ percibidos tienen un impacto positivo en la CS por segmento, es decir, una correlación lineal $r>0.7$.

\section{Metodología}

\section{Diseño de la investigación}

De acuerdo con la clasificación de la Cámara Nacional de la Industria de Restaurantes y Alimentos Sazonados (CANIRAC, Monroy-Ceseña y Urcádiz-Cázares, 2019), se consideraron los restaurantes especializados en comida Italiana, Mexicana, Japonesa, de Mariscos e Internacional en el destino turístico de Todos Santos, México.

La población de estudio se consideró como los comensales mayores de 18 años clasificados dentro de las especialidades descritas anteriormente. En esta investigación, definimos turistas nacionales como los comensales que viven en México, y turistas foráneos como comensales que viven en otro país. Las encuestas se realizaron durante el verano del 2018 (de junio a agosto), por lo que se trata de un estudio observacional transversal. El número y la variación de personas en esta población es desconocida, sin embargo, un grupo focal con personal y gerencia estimó una fluctuación de 6,885 personas por semana (ver tabla 1 para la focalización de especialidad).

Sobre este patrón, se tomó una muestra aleatoria de 162 comensales para encuestar con un instrumento de 26 ítems basado en las dimensiones propuestas y adaptadas por Monroy-Ceseña y Urcádiz-Cázares (2019). Las dimensiones evaluadas fueron las Instalaciones

\footnotetext{
"Visión de Futuro" Año 17, Volumen No 24 N², Julio - Diciembre 2020 - Pág 194 - 210

URL de la Revista: http://visiondefuturo.fce.unam.edu.ar/index.php/visiondefuturo/index

URL del Documento: https://visiondefuturo.fce.unam.edu.ar/index.php/visiondefuturo/issue/view/18

ISSN 1668 - 8708 - Versión en Línea

E-mail: revistacientifica@fce.unam.edu.ar
} 
(infraestructura), la Accesibilidad, el Capital Humano, la Atmósfera y los Alimentos, y asumimos igual peso en las dimensiones. Este instrumento permite medir la percepción promedio de la calidad del servicio (SQ), como la satisfacción del cliente (CS) por dimensiones. La encuesta mide las actitudes basadas en la Escala Likert (Hernández-Sampieri, Fernández-Collado y Baptista, 2014), que consiste en un cierto número de ítems (o preguntas) donde cada ítem se valora en la misma escala continua que relaciona dos categorías ordinales, como un enfoque robusto. La encuesta utilizó la siguiente escala: el valor 7 representa Totalmente de acuerdo y el valor 1 representa Totalmente en desacuerdo (sin ninguna categoría dentro de la escala). Además, implementamos un espacio libre para comentarios sobre el servicio global y la satisfacción experimentada en el restaurante. Debido a que se supone que la variable es continua, un tamaño de muestra de al menos 30 sería suficiente para justificar el teorema del límite central (Anderson et al., 2018) y hacer estimaciones de la media de cada dimensión del constructo. En este estudio, se obtuvo una muestra aleatoria de 162 comensales (de 49 restaurantes), que es adecuada para el tamaño estimado de la población.

\section{Encuesta piloto, fiabilidad y validez de datos.}

Una vez que se diseñaron las escalas de encuesta y valoración para la $S Q$ y CS, el siguiente paso fue explorar la consistencia interna de la encuesta (Hernández-Sampieri, Fernández-Collado \& Baptista, 2014). En consecuencia, se realizó una muestra piloto de 15 encuestas (tres por especialidad) para establecer la consistencia interna mediante la estimación del alfa de Cronbach (Bonnet, 2002):

$$
\alpha=\left[\frac{k}{k-1}\right]\left[1-\frac{\sum_{i=1}^{k} s_{i}^{2}}{s_{t}^{2}}\right]
$$

Donde Alpha de Cronbach, $\mathrm{k}=$ número de ítems, $s_{i}^{2}==$ varianza del ítem $i, s_{t}^{2}=$ varianza de la suma de todos los ítems. Este estadístico se utiliza para demostrar la validez y fiabilidad de las encuestas basadas en la escala Likert. Un alfa de Cronbach se valora entre 0 y 1 , donde los valores superiores a 0,70 (o 70\%) se interpretan como un instrumento de medición aceptable (Hernández-Sampieri, Fernández-Collado y Baptista, 2014). El resultado obtenido para definir la confiabilidad del instrumento que se utilizó para esta investigación fue 0.90, que a discreción de Celina y Campo (2005), este valor manifiesta la consistencia interna, es decir,

\footnotetext{
"Visión de Futuro" Año 17, Volumen No 24 N², Julio - Diciembre 2020 - Pág 194 - 210

URL de la Revista: http://visiondefuturo.fce.unam.edu.ar/index.php/visiondefuturo/index

URL del Documento: https://visiondefuturo.fce.unam.edu.ar/index.php/visiondefuturo/issue/view/18

ISSN 1668 - 8708 - Versión en Línea

E-mail: revistacientifica@fce.unam.edu.ar
} 
muestra la correlación entre cada una de las preguntas por dimensión; un valor superior a 0,70 revela una fuerte relación entre las preguntas.

Según Tristan (2008), para validar un instrumento, propone un modelo de organización de un panel de evaluación, compuesto por especialistas en la tarea a evaluar. De esta manera, en el proceso de validación de contenido para este trabajo de investigación, fueron necesarias dos tareas; el primero de ellos, una revisión de literatura especializada, y el juicio de expertos. Tres profesores con un título de doctor en ciencias económicas y administrativas y dos administradores de restaurantes revisaron y validaron la encuesta.

Tabla $N^{\circ}$ 1. Distribución de encuestas en restaurantes por especialidad. La población es una medida aproximada en el grupo focal (personas que pagan por su consumo por asistencia)

\begin{tabular}{lcccc}
\hline ESPECIALIDAD & $\begin{array}{c}\text { TOTAL DE } \\
\text { RESTAURANTES }\end{array}$ & $\begin{array}{c}\text { RESTAURANTES } \\
\text { EVALUADOS }\end{array}$ & $\begin{array}{c}\text { TAMAÑO DE } \\
\text { POBLACIÓN }\end{array}$ & $\begin{array}{c}\text { TAMAÑO DE } \\
\text { MUESTRA }\end{array}$ \\
\hline Mexicana & 31 & 6 & 4,200 & 39 \\
Italiana & 5 & 4 & 300 & 25 \\
Japonesa & 3 & 3 & 225 & 32 \\
Mariscos & 8 & 4 & 1,440 & 33 \\
Internacional & 12 & 4 & 720 & 33 \\
Total & 49 & 31 & 6,885 & 162 \\
\hline
\end{tabular}

Fuente: Elaboración Propia

\section{Análisis estadístico}

Para evaluar la SQ y CS, los datos se procesaron a través del software IBM SPSS Statistics versión 23 para calcular las medias de cada dimensión y especialidades. Se realizó una serie de pruebas $t$ de dos muestras (sin suponer varianzas iguales, Anderson et al., 2018) bajo las hipótesis previamente indicadas. Las pruebas se realizaron bajo el supuesto de normalidad y utilizando un nivel de significancia del 5\% (alfa $=0.05)$. Esto permite evaluar si una especialidad de restaurante en cada dimensión difiere significativamente entre la percepción media turística nacional y foránea. Para comparar las dimensiones de los restaurantes en general, realizamos un análisis de varianza unidireccional (ANOVA) utilizando las medias de las dimensiones de la QS y la CS (como factor) por segmento. Para calcular el análisis, utilizamos un nivel de significancia del $5 \%$ (alfa $=0.05$ ) y asumimos varianzas desiguales (Anderson et al., 2018) donde se habilitó la prueba de Tukey por defecto.

\footnotetext{
"Visión de Futuro" Año 17, Volumen N²4 N², Julio - Diciembre 2020 - Pág 194 - 210

URL de la Revista: http://visiondefuturo.fce.unam.edu.ar/index.php/visiondefuturo/index

URL del Documento: https://visiondefuturo.fce.unam.edu.ar/index.php/visiondefuturo/issue/view/18

ISSN 1668 - 8708 - Versión en Línea

E-mail: revistacientifica@fce.unam.edu.ar
} 
Suponiendo que las medias de cada especialidad de la SQ como variable independiente y la CS como variable dependiente, se realizó un análisis de correlación lineal de Pearson para comparar la media de la CS de las percepciones de SQ, considere las cinco especialidades dadas a cada dimensión ( $\mathrm{n}=25)$ por segmentos. Los resultados fueron contrastados por el valor 0.7 de acuerdo con Anderson et al. (2018).

\section{Resultados y discusiones}

Los valores promedio de la calidad del servicio (SQ) y la satisfacción del cliente (CS) percibidos por los comensales nacionales y foráneos se muestran en las Tablas 2 y 3 , respectivamente. Los comensales nacionales percibieron la SQ de 5.36 a 6.47 como valores medios extremos tomando en cuenta todas las especialidades y dimensiones (Tabla 2). Del mismo modo, los comensales foráneos percibieron la SQ de 5.64 a 6.58. Según la prueba de hipótesis, todas las dimensiones de la especialidad italiana percibidas por los turistas nacionales son significativamente diferentes $(p<0.05)$ de las foráneas. Particularmente, la dimensión de accesibilidad de los restaurantes japoneses tuvo una diferencia significativa. El resto de los valores se mantienen similares sobre SQ. Con respecto a la CS, los comensales nacionales percibieron un promedio de 5.53 a 6.67, mientras que los comensales foráneos percibieron de 5.66 a 6.82 (Tabla 3). Cuatro dimensiones (excepto instalaciones) de la CS de la especialidad italiana fueron significativamente diferentes. Dos dimensiones (instalaciones y alimentos) de la especialidad japonesa dieron como resultado diferencias significativas entre las percepciones por segmento (Tabla 3). Con respecto a la hipótesis $\mathrm{H} 1$, los resultados sugieren una aceptación parcial del postulado.

A pesar de las diferencias socioculturales y económicas de los segmentos en comparación, los resultados revelan que la $S Q$ y la CS de los restaurantes en la ciudad mágica de Todos Santos se han valorado de manera similar, excepto los restaurantes de especialidades italianas y parcialmente de especialidad japonesa. En esta excepción y con la información disponible, es difícil determinar con precisión la razón por la cual los comensales foráneos perciben tanto la SQ como la CS con mayor valor. Algunos comentarios libres sobre esta especialidad hechos por comensales nacionales mencionan que las características del sabor $(n=5)$, el precio $(n=3)$ y el tamaño de la porción $(n=3)$ son generalmente razones para mejorar para el restaurante donde se ofreció el servicio. Estas características podrían intervenir

\footnotetext{
"Visión de Futuro" Año 17, Volumen No 24 N², Julio - Diciembre 2020 - Pág 194 - 210

URL de la Revista: http://visiondefuturo.fce.unam.edu.ar/index.php/visiondefuturo/index

URL del Documento: https://visiondefuturo.fce.unam.edu.ar/index.php/visiondefuturo/issue/view/18

ISSN 1668 - 8708 - Versión en Línea

E-mail: revistacientifica@fce.unam.edu.ar
} 
en la valoración media de la especialidad. En contraste, los comentarios libres de los comensales foráneos $(n=4)$ fueron relativos a la distinción de excelente o buen servicio.

Tabla $\mathbf{N}^{\circ}$ 2. Comparación promedio de la calidad del servicio percibido (SQ) por los turistas (comensales) nacionales y foráneos en los restaurantes de Todos Santos. Especialidades de comida: MEX=Mexicana, ITA=Italiana, JAP=Japonesa, SEA=mariscos e INT=Internacional. Diferencia significativa entre medias $(p<0.05)$ desarrolladas con la prueba $t$ de dos muestras*

\begin{tabular}{|c|c|c|c|c|c|c|c|c|c|c|}
\hline & \multicolumn{5}{|c|}{ TURISTAS NACIONALES } & \multicolumn{5}{|c|}{ TURISTAS FORÁNEOS } \\
\hline & MEX & ITA & JAP & SEA & INT & MEX & ITA & JAP & SEA & INT \\
\hline Instalaciones & 6.28 & $5.69^{\star}$ & 5.62 & 5.67 & 5.94 & 6.26 & $6.37^{*}$ & 5.70 & 5.91 & 6.22 \\
\hline Acesibilidad & 6.22 & $5.80^{\star}$ & $5.97^{\star}$ & 5.64 & 6.42 & 6.21 & $6.40^{*}$ & $6.39^{\star}$ & 5.70 & 6.14 \\
\hline Capital humano & 6.25 & $5.36^{\star}$ & $6.04^{*}$ & 5.58 & 6.47 & 6.25 & $6.54^{\star}$ & 6.35 & 5.64 & 6.20 \\
\hline Atmósfera & 6.26 & $5.65^{\star}$ & 6.10 & 5.98 & 6.35 & 6.39 & $6.36^{*}$ & 6.29 & 5.83 & 6.28 \\
\hline Alimentos & 6.18 & $6.08^{\star}$ & 6.06 & 6.43 & 6.32 & 6.18 & $6.58^{*}$ & 6.07 & 6.38 & 6.46 \\
\hline Media & 6.24 & $5.72^{\star}$ & 500 & 5.86 & 6.30 & 6.26 & $6.45^{\star}$ & 6.16 & 5.89 & 6.26 \\
\hline
\end{tabular}

Fuente: Elaboración Propia

Con respecto a la hipótesis $\mathrm{H} 2$ sobre la comparación de las percepciones de dimensión para cada segmento, el análisis de varianza (factor unidireccional ANOVA) muestra que hay suficiente evidencia estadística para respaldar que no hay una diferencia significativa entre el valor promedio de las dimensiones bajo un nivel de confianza de $95 \%(p<0.05)$ tanto la $S Q$ como SC para cada segmento. Este resultado nos permite rechazar la hipótesis H2 y aclarar que el valor promedio de las dimensiones de los alimentos y del capital humano no es más alto que el resto de las dimensiones. Un punto destacado en este análisis es que se supone que cada dimensión tiene el mismo peso. Según Dagger y Sweeny (2007), la ponderación de las dimensiones no debe considerarse por igual, en este caso, en la industria de los restaurantes, la dimensión de los alimentos podría tener más peso (o importancia) que la accesibilidad. En este estudio, no implementamos una ponderación diferente para permitir la comparación en el ANOVA, aunque, para estudios futuros, los datos están disponibles para una ponderación y posibles comparaciones.

Tabla $N^{\circ}$ 3. Comparación promedio de satisfacción del cliente percibida (CS) por los turistas (comensales) nacionales y foráneos en los restaurantes de Todos Santos. Especialidades de comida: MEX=Mexicana, ITA=Italiana, JAP=Japonesa, SEA=mariscos e INT=Internacional. Diferencia significativa entre medias $(p<0.05)$ desarrolladas con la prueba $t$ de dos muestras*

\begin{tabular}{llllllllll}
\hline \multicolumn{4}{c}{ TURISTAS NACIONALES } & \multicolumn{4}{c}{ TURISTAS FORÁNEOS } \\
\hline MEX & ITA & JAP & SEA & INT & MEX & ITA & JAP & SEA & INT
\end{tabular}

\footnotetext{
"Visión de Futuro" Año 17, Volumen No 24 N², Julio - Diciembre 2020 - Pág 194 - 210

URL de la Revista: http://visiondefuturo.fce.unam.edu.ar/index.php/visiondefuturo/index

URL del Documento: https://visiondefuturo.fce.unam.edu.ar/index.php/visiondefuturo/issue/view/18

ISSN 1668 - 8708 - Versión en Línea

E-mail: revistacientifica@fce.unam.edu.ar
} 


\begin{tabular}{lllllllllll} 
Instalaciones & 6.47 & 6.13 & $\mathbf{5 . 6 7}^{*}$ & 5.67 & 6.58 & 6.41 & 6.36 & $\mathbf{6 . 3 6}$ & 5.96 & 6.10 \\
Acesibilidad & 6.41 & $\mathbf{5 . 6 0}^{*}$ & 6.33 & 5.83 & 6.17 & 6.23 & $\mathbf{6 . 7 0 ^ { * }}$ & 6.55 & 5.58 & 6.33 \\
Capital humano & 6.24 & $\mathbf{5 . 6 0}^{*}$ & 6.29 & 5.58 & 6.50 & 6.55 & $\mathbf{6 . 6 0}^{\star}$ & 6.55 & 5.66 & 6.29 \\
Atmósfera & 6.59 & $\mathbf{5 . 5 3}^{*}$ & 6.14 & 5.92 & 6.58 & 6.36 & $\mathbf{6 . 8 0}^{\star}$ & 6.27 & 5.76 & 6.57 \\
Alimentos & 6.06 & $\mathbf{6 . 5 3}^{\star}$ & $\mathbf{5 . 8 1}^{*}$ & 6.67 & 6.42 & 6.45 & $\mathbf{6 . 4 0}^{\star}$ & $\mathbf{6 . 8 2}$ & 6.58 & 6.76 \\
Media & 6.35 & 5.88 & 6.04 & 5.93 & 6.45 & 6.40 & 6.56 & 6.51 & 5.91 & 6.41 \\
\hline
\end{tabular}

Fuente: Elaboración Propia

Algunos trabajos relacionados con la SQ y la CS sugieren que las dimensiones de los alimentos son el factor principal en el servicio percibido. Namin (2017) cita en su investigación que el principal determinante significativo en la calidad del servicio percibido por los comensales es la comida. Según sus principales hallazgos, la oferta de un menú saludable y acompañado de sabrosas comidas, se convierten en criterios de enorme relevancia en la búsqueda de la satisfacción de los comensales en la industria. Para la industria de la comida rápida, uno de los factores de mayor atención en cualquier proceso de servicio está relacionado con el capital humano. Según Voon (2012) en su estudio sobre la calidad del servicio en restaurantes de comida rápida, uno de los principales factores que afectan la percepción de los clientes es la influencia del capital humano en su interacción continua con los clientes, así como el precio de venta asignado a la oferta de comida. En contraste, esta investigación muestra que la dimensión de alimentos y del capital humano no son los factores más altos en la percepción de la SQ y de la CS que ofrecieron las personas en los restaurantes de la ciudad mágica de Todos Santos. En este sentido, las dimensiones en su totalidad se percibieron de manera similar (dejando de lado que no se trata de comida rápida).

De acuerdo con la hipótesis H3, sobre el impacto de la SQ en SC, el coeficiente de correlación lineal de Pearson fue $r=0.80$ para la percepción turística nacional y $r=0.72$ para la percepción turística foránea. Siguiendo a Celina y Campo (2005), Hernández-Sampieri, Fernández-Collado y Baptista (2014) y Anderson et al. (2018), estos valores indican una correlación positiva moderada entre ambas variables, en este caso, las percepciones hechas por los comensales en las diferentes especialidades en cada dimensión. En este sentido, la correlación matemáticamente sugiere una incidencia o un impacto positivo de la SQ sobre SC, aunque no necesariamente esté enraizado estrictamente en una causalidad, como lo señalan Sureshchandar, Rajendran y Anatharaman (2002) y Anderson et al. (2018) El discernimiento entre el plano causal y el matemático con esta técnica estadística es limitado, en este sentido,

\footnotetext{
"Visión de Futuro" Año 17, Volumen No 24 N², Julio - Diciembre 2020 - Pág 194 - 210

URL de la Revista: http://visiondefuturo.fce.unam.edu.ar/index.php/visiondefuturo/index

URL del Documento: https://visiondefuturo.fce.unam.edu.ar/index.php/visiondefuturo/issue/view/18

ISSN 1668 - 8708 - Versión en Línea

E-mail: revistacientifica@fce.unam.edu.ar
} 
se recomienda realizar estudios más específicos que ayuden a comprender la posible relación causal entre ambos constructos. Sin embargo, algunos trabajos informan esta tendencia (Sureshchandar, Rajendran y Anatharaman, 2002; Muhamad, Hashim y Rozila, 2016) incluso para la misma región de estudio (Monroy-Ceseña y Urcádiz-Cázares, 2019). Los resultados de este trabajo de investigación se pueden utilizar para guiar a las empresas a mantener y mejorar sus servicios en el pueblo mágico de Todos Santos.

\section{CONCLUSIÓN}

Este trabajo es un punto de partida para el análisis estratégico de restaurantes en el destino turístico del pueblo mágico de Todos Santos, México. De manera inédita, se presenta la percepción de la calidad del servicio (SQ) y la satisfacción del cliente (CS) por segmento, entre turistas nacionales y foráneos, lo que contribuye tanto con las investigaciones futuras como con información para el desarrollo de los restaurantes.

Los resultados muestran que tanto el SQ como el CS percibidos entre los comensales nacionales y foráneos son similares, a excepción de la comida italiana y parcialmente japonesa. En este rubro, la hipótesis $\mathrm{H} 1$ es parcialmente aceptada. Por otro lado, suponiendo que la dimensión de los alimentos y el capital humano debe ser el principal determinante en la percepción del servicio y la satisfacción del comensal, nuestros resultados muestran que esta dimensión no es la principal (la más valorada en promedio). El análisis de varianza mantiene que entre las dimensiones no hay una diferencia significativa. Esta percepción fue consistente tanto en los comensales nacionales como en foráneos. En este sentido, la hipótesis H2 es rechazada. Finalmente, el análisis de correlación indica que el SQ percibido tiene un impacto positivo moderado en el CS en ambos segmentos encuestados, aunque no debería tener una relación causal lineal estrictamente.

\section{REFERENCIAS}

Aeker, D., \& Jacobson, R. (1994). The financial information content of perceived quality. Journal of Marketing Research, 31(2), 191-201.https://www.jstor.org/stable/3152193

\footnotetext{
"Visión de Futuro" Año 17, Volumen No 24 N², Julio - Diciembre 2020 - Pág 194 - 210

URL de la Revista: http://visiondefuturo.fce.unam.edu.ar/index.php/visiondefuturo/index

URL del Documento: https://visiondefuturo.fce.unam.edu.ar/index.php/visiondefuturo/issue/view/18

ISSN 1668 - 8708 - Versión en Línea

E-mail: revistacientifica@fce.unam.edu.ar
} 
Akbaba, A. (2006). Measuring service quality in the hotel industry: A study in a business hotel in Turkey. International Journal of Hospitality Management, 25(2), 170-192. https://doi.org/10.1016/.j.jhm.2005.08.006

Anderson, D. R., Sweeney, D. J., Williams, T. A., Camm, J. F., \& Cochran, J. J. (2018). Statistics for business \& economics. USA Cengage Learning.

Armstrong, K. (2012). Principles of Marketing (14th edition). New Jersey: USA Pearson Education Inc.

Aurier, P., Evrard, Y., \& N'Goala, G. (2004). Comprendre et mesurer la valeur du point de vue du consommateur. Recherche et Applications en Marketing, 19(3), 1-20. Obtenido de https://doi.org/10.1177/076737010401900301

Bonnet, D. G. (2002). Sample size requirements for testing and estimating coefficient alpha. Journal of Education and Behavioral Statistics, 27(335), 335-340. https://doi.org/10.3102/10769986027004335

Buttle, F. (1996). "SERVQUAL: review, critique, research agenda", European Journal of Marketing, Vol. 30 No. 1, pp. 8-32. https://doi.org/10.1108/03090569610105762

Celina, H., \& Campo, A. (2005). Aproximación al uso del coeficiente alfa de cronbach. Revista Colombiana de Psiquiatría, 34(4), 572-580.

Cronin, J., \& Taylor, S. A. (1994). SERVPERF vs SERVQUAL: Reconciling performance-based and perceptions-minus expectations measurement of service quality. Journal of Marketing, 58(1), 125-131.

Dagger, T. S. \& Sweeney, J. C. (2007). Service quality attribute weights: how do novice and longer-term customer construct service quality perceptions? Journal of Service Reseach, 10, 22-42. https://doi.org/10.1177/1094670507303010

Evrard, Y., \& Aurier, P. (1996). Identification and validation of the components of the personobject relationship. Journal of Business Research, 37(2), 127-134. https://doi.org/10.1016/0148-2963(96)00054-9

Fujun, L., Hutchinson, J., Li, D., \& Changhong, B. (2007). An empirical assessment and application on SERVQUAL in mainland china's mobile communications industry. The International Journal of Quality \& Reliability Management, 24(3), 244-262. https://doi.org/10.1108/02656710710730852

\footnotetext{
"Visión de Futuro" Año 17, Volumen No 24 N 2, Julio - Diciembre 2020 - Pág 194 - 210

URL de la Revista: http://visiondefuturo.fce.unam.edu.ar/index.php/visiondefuturo/index

URL del Documento: https://visiondefuturo.fce.unam.edu.ar/index.php/visiondefuturo/issue/view/18

ISSN 1668 - 8708 - Versión en Línea

E-mail: revistacientifica@fce.unam.edu.ar
} 
Gardial, F. S., Clemons, S. D., Woodruff, B., Schumann, W. D., \& Burns, J. (1994). Comparing consumer's recall of pre-purchase and post-purchase product evaluation experience. Journal of Consumer Research, 20, 548-560. https://www.jstor.org/stable/2489758

Gilbert, G. R., \& Veloutsou, C. (2006). A cross-industry comparison of customer satisfaction. The Journal of Service Marketing, 20(5), 298-308. https://doi.org/10.1108/08876040610679918

Gilbert, G. R., Veloutsou, C., Goode, M., \& Moutinho, L. (2004). Measuring customer satisfaction in the fast food industry: a cross national approach. Journal of Service Marketing, 18(5), 371-383. https://doi.org/10.1108/08876040410548294

Guzmán-López, A., \& Cárcamo-Solís, M. L. (2014). La evaluación de la calidad en el servicio: caso de estudio "Restaurant Familiar Los Fresnos". Acta Universitaria, 24(3), 35-49.

Halstead, D., Hartman, D., \& Schmidt, S. L. (1994). Multisource effects on the satisfaction formation process. Journal of the Academy of Marketing Science, 22(2), 114-129. https://doi.org/10.1177/0092070394222002

Hernándes-de-Velazco, J., Chumaceiro, A., \& Atencio, E. (2009). Calidad de servicio y recurso humano: caso de estudio tienda por departamentos. Revista Venezolana de Gerencia (RVG), 14(47), 457-470.

Hernández-Sampieri, R., Fernández-Collado, C., \& Baptista, L. (2014). Metodología de la Investigación. México: McGraw Hill Education.

Kim, O., Seo, S., \& Nurhidayati, V. (2019). Scale to measure tourist value of destination restaurant service. International Journal of Contemporary Hospitality Management, 31(7), 2827-2844. https://doi.org/10.1108//JCHM-05-2018-0443

Kotler, P., \& Armstrong, G. (2012). Principles of Marketing. Pearson.

Ladhari, R. (2008). Alternatives measures of service quality: a review. Managing Service Quality, 18(1), 65-86. https://doi.org/10.1108/09604520810842849

Ladhari, R. (2009). A review of twenty years of SERVQUAL research. International Journal of $\begin{array}{lll}\text { Quality } \quad \text { and Service } \quad \text { Sciences, } & 1(2), & \text { 172-198. }\end{array}$ https://doi.org/10.1108/17566690910971445

Mano, H., \& Oliver, R. (1993). Assessing the dimensionality and structure of the consumption experience: Evaluating, feeling, and satisfaction. Journal of Consumer Research, 20, 451-466. https://doi.org/10.1086/209361

\footnotetext{
"Visión de Futuro" Año 17, Volumen No 24 N², Julio - Diciembre 2020 - Pág 194 - 210

URL de la Revista: http://visiondefuturo.fce.unam.edu.ar/index.php/visiondefuturo/index

URL del Documento: https://visiondefuturo.fce.unam.edu.ar/index.php/visiondefuturo/issue/view/18

ISSN 1668 - 8708 - Versión en Línea

E-mail: revistacientifica@fce.unam.edu.ar
} 
Moliner-Cantos, C. (2001). Calidad de servicio y satisfacción del cliente. Revista de Psicología del Trabajo y de las Organizaciones, 17(2), 233-235.

Monroy-Ceseña, M. A, \& Urcádiz-Cázares, F. J. (2019). Calidad en el servicio y su incidencia en la satisfacción del comensal en restaurantes de La Paz, México. Investigación Administrativa, 48, 123 https://doi.org/10.35426/IAv48n123.06

Muhamad, O., Hashim, A., \& Rozila, A. (2016). Service Quality, Customers' Satisfaction and the Moderating Effects of Gender: A Study of Arabic Restaurants. Procedia - Social and Behavioral Sciences, 224, 384-392. https://doi.org/10.1016/i.sbspro.2016.05.393

Namin, A. (2017). Revisiting customer's perception of service quality in fast-food restaurants. Journal of Retailing and Consumer Services, 34, 70-81. https://doi.org/10.1016/j.jretconser.2016.09.008

Namkung, Y., Cheong S. J. \& Choi, S. K. (2011). Customer complaints in restaurants: Do they differ by service stages and loyalty levels? International Journal of Hospitality Management, 30(3):495-502. https://doi.org/10.1016/j.ijhm.2010.07.005

Nguyen, Q., Nisar, T., Knox, D., \& Prabhakar, G. (2019). Understanding customer satisfaction in the UK quick service restaurant industry: The influence of the tangible attributes of perceived service quality, British Food Journal, 120(6), 1207-1222. https://doi.org/10.1108/BFJ-08-2017-0449

Oliver, R. L., Rust, R. T., \& Varki, S. (1997). Customer Delight: foundations, findings and managerial insight. Journal of Retailing, 73(3), 311-336. https://doi.org/10.1016/s00224359(97)90021-X

Rodríguez-Herrera, I. M., Pulido-Fernández, J. I., Vargas-Vázquez, A., \& Shaadi-Rodríguez, R. M. A. (2018). Dinámica relacional en los pueblos mágicos de México. Estudio de las implicaciones de la política turística a partir del análisis de redes. Turismo y Sociedad, (22), 85-104. https://doi.org/10.18601/01207555.n22.05

Ryu, K., \& Han, H. (2011). New or repeat customers: how does physical environment influence their restaurant experience? International Journal of Hospitality Management, 30(3), 599-611. https://doi.org/10.1016/j.ijhm.2010.11.004

Ryu, K., Lee, H., \& Gon-Kim, W. (2012). The influence of the quality of the physical environment, food, and service on restaurant image, customer perceived value, customer satisfaction,

\footnotetext{
"Visión de Futuro" Año 17, Volumen No 24 N 2, Julio - Diciembre 2020 - Pág 194 - 210

URL de la Revista: http://visiondefuturo.fce.unam.edu.ar/index.php/visiondefuturo/index

URL del Documento: https://visiondefuturo.fce.unam.edu.ar/index.php/visiondefuturo/issue/view/18

ISSN 1668 - 8708 - Versión en Línea

E-mail: revistacientifica@fce.unam.edu.ar
} 
and behavioral intentions. International Journal of Contemporary Hospitality Management, 24(2), 200-223. https://doi.org/10.1108/09596111211206141

Sartor, M. (2019). Customer Satisfaction Analyses, In: Sartor, M. and Orzes, G. (Ed.) Quality Management: Tools, Methods, and Standards, Emerald Publishing Limited, pp. 109-116. https://doi.org/10.1108/978-1-78769-801-720191007

Spreng, R. A., \& Olshavsky, R. W. (1993). A desires congruency model of costumer satisfaction.

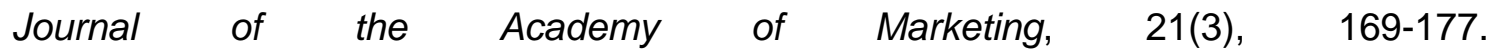
http://dx.doi.org/10.1177/0092070393213001

Spreng, R. A., MacKenzie, S. B., \& Olshavsky, R. W. (1996). A reexamination of the determinants of customer satisfaction. Journal of Marketing, 60, 15-32. http://dx.doi.org/10.2307/1251839

Sureshchandar, G. S., Rajendran, C., \& Anatharaman, R. N. (2002). The relationship between service quality and customer satisfaction - a factor specific approach. Journal of Services Marketing, 16(4), 363-379. https://doi.org/10.1108/08876040210433248

Tristan, A. (2008). Modificación al modelo de Lawshe para el dictamen cuantitativo de la validez de contenido de un instrumento objetivo. Avances en Medición, 6, 37 - 48.

Vanhamme, J. (2002). La satisfaction des consommateurs spécifique à une transaction: définition, antécédents, mesures et modes. Recherche et Applications en Marketing, 17(2), 55-85. https://www.jstor.org/stable/40589322

Vera, J., \& Trujillo, A. (2017). Escala Mexicana de Calidad en el Servicio (EMCASER). Innovar, 27(63), 43 - 59. https://doi.org/10.15446/innovar.v26n63.60665

Voon, B. H. (2012). Role of service environment for restaurants: the youth customer's perspective. Procedia-Social and Behavioral Sciences (38), 388-395. https://doi.org/10.1016/i.sbspro.2012.03.361

Westbrook, R. (1987). Product/Consumption-based affective responses and postpurchase processes. Journal of Marketing Research, 24, 258-270.

Wilkins, H., Merrilees, B., \& Herington, C. (2007). Towards an understanding of total service quality in hotels. International Journal of Hospitality Management, 26(4), 840-853. https://doi.org/10.1016/j.ijhm.2006.07.006

Yi, Y. (1991). A Critical Review of Consumer Satisfaction. In V. A. Zeithaml (Ed.), Review of Marketing 1990 (pp. 68-123). Chicago, IL: American Marketing Association.

\footnotetext{
"Visión de Futuro" Año 17, Volumen No 24 N², Julio - Diciembre 2020 - Pág 194 - 210

URL de la Revista: http://visiondefuturo.fce.unam.edu.ar/index.php/visiondefuturo/index

URL del Documento: https://visiondefuturo.fce.unam.edu.ar/index.php/visiondefuturo/issue/view/18

ISSN 1668 - 8708 - Versión en Línea

E-mail: revistacientifica@fce.unam.edu.ar
} 
Zairi, M. (2000). Managing Customer Dissatisfaction Though Effective Complaint Management $\begin{array}{llll}\text { Systems. The } & \text { 331-337. }\end{array}$ https://doi.org/10.1108/09544780010341932

Zárraga, L., Molina, V., \& Corona, E. (2018). La satisfacción del cliente basada en la calidad en el servicio a través de la eficiencia del personal y eficiencia del servicio: un estudio empírico de la industria restaurantera. Revista de Estudios en Contaduría, Administración e Informática, 7(18), 46-65.

\section{RESUMEN BIOGRAFICO}

\section{Mauro Alejandro Monroy Ceseña}

Profesor - Investigador del departamento académico de Economía en la Universidad Autónoma de Baja California Sur, con doctorado en Ciencias Administrativas en el Instituto de Estudios Universitarios de Puebla.

\section{Francisco Javier Urcádiz Cázares}

Doctor en Ciencias Marinas (2018), dedicado a la docencia en el área de Ciencias Básicas del Tecnológico Nacional de México, con intereses multidisciplinarios en el diseño y la aplicación de métodos estadísticos.

\footnotetext{
"Visión de Futuro" Año 17, Volumen No 24 N², Julio - Diciembre 2020 - Pág 194 - 210

URL de la Revista: http://visiondefuturo.fce.unam.edu.ar/index.php/visiondefuturo/index

URL del Documento: https://visiondefuturo.fce.unam.edu.ar/index.php/visiondefuturo/issue/view/18

ISSN 1668 - 8708 - Versión en Línea

E-mail: revistacientifica@fce.unam.edu.ar
} 